\title{
Overstuffing in resurfacing hemiarthroplasty is a potential risk for failure
}

\author{
Pieter C. Geervliet ${ }^{1 *}$, Jore H. Willems ${ }^{1}$, Inger N. Sierevelt ${ }^{2}$, Cornelis P. J. Visser ${ }^{3}$ and Arthur van Noort $^{2}$
}

\begin{abstract}
Purpose: Literature describes the concern of an overstuffed shoulder joint after a resurfacing humeral head implant $(\mathrm{RHHI})$. The purpose of this study was to evaluate inter-observer variability of (1) the critical shoulder angle (CSA), (2) the length of the gleno-humeral offset (LGHO), and (3) the anatomic center of rotation (COR) in a patient population operated with a Global Conservative Anatomic Prosthesis (CAP) RHHI. The measurements were compared between the revision and non-revision groups to find predictive indicators for failure.

Methods: Pre- and postoperative radiographs were retrieved from 48 patients who underwent RHHI from 2007 to 2009 using a Global CAP hemiarthroplasty for end-stage osteoarthritis. This cohort consisted of 36 females (12 men) with a mean age of 77 years (SD 7.5). Two musculoskeletal radiologist and two specialized shoulder orthopedic surgeons measured the CSA, LGHO, and COR of all patients.

Results: The inter-observer reliability showed excellent reliability for the CSA, LGHO, and the COR, varying between 0.91 and 0.98. The mean COR of the non-revision group was $4.9 \mathrm{~mm}$ (SD 2.5) compared to mean COR of the revision group, $8 \mathrm{~mm}(\mathrm{SD} 2.2)(p<0.01)$. The COR is the predictor of failure (OR $1.90(95 \% \mathrm{Cl} 1.19-3.02))$ with a cut of point of $5.8 \mathrm{~mm}$. The mean CSA was $29.8^{\circ}$ (SD 3.9) There was no significant difference between the revision and non-revision groups $(p=0.34$ ). The mean LGHO was $2.6 \mathrm{~mm}$ (SD 3.3) post-surgery. The mean LGHO of the revision group was 3.9 (SD 1.7) $(p=0.04)$ post-surgery. Despite the difference in mean LGHO, this is not a predictor for failure.

Conclusion: The CSA, LGHO, and COR can be used on radiographs and have a high inter-observer agreement. In contrast with the CSA and LGHO, we found a correlation between clinical failure and revision surgery in case of a deviation of the COR greater than $5 \mathrm{~mm}$.
\end{abstract}

Trial registration: Institutional review board, number: ACLU 2016.0054, Ethical Committee number: CBP M1330348. Registered 7 November 2006.

Keywords: Resurfacing humeral head implant, Overstuffing, Shoulder, Radiographs, Revision

\section{Introduction}

The hemi resurfacing humeral head implant (RHHI) provides good clinical results for patients with gleno-humeral osteoarthritis [1-7]. The purpose of a RHHI is to restore the patient's individual anatomy and the lateral offset of

\footnotetext{
*Correspondence: geervliet@gmail.com

${ }^{1}$ NoordWest Clinics, Department of Orthopedic Surgery, Shoulder Unit, Wilhelminalaan 12, 1815, JD, Alkmaar, the Netherlands

Full list of author information is available at the end of the article
}

the proximal humerus while preserving the bone stock of the humeral head [8-10].

Sizing of the proximal humerus is generally preoperative estimated on the radiograph and definitely measured during surgery. Because of a deformed proximal humerus, surgeons often have difficulty to accurate assess the correct size of the implant and restoring the anatomy compared with stemmed arthroplasty $[8,10]$. In literature, high rate of revision of the RHHI is a concern [11-13]. Alolabi et al. [8] found a possible relation with

(c) The Author(s). 2019 Open Access This article is distributed under the terms of the Creative Commons Attribution 4.0 International License (http://creativecommons.org/licenses/by/4.0/), which permits unrestricted use, distribution, and 
overstuffing; however, in literature, there is no definite correlation reported between overstuffing and revision.

This study was performed as an extension to an ongoing follow-up study in patients treated with uncemented Global Conservative Anatomic Prosthesis (CAP) (DePuy Synthes, Warsaw, IN, USA) hemi resurfacing shoulder prosthesis from 2007 until 2009 [13-16]. At the 5-8 years follow-up, our results are in line with other studies of a concerning high rate of revision $[13,16]$.

The aim of this current radiographic study was to evaluate the ability to restore humeral head anatomy and to determine the inter-observer reliability of the critical shoulder angle (CSA), length of the glenohumoral offset (LGHO), and deviation of the center of rotation (COR) in a hemi RHHI.

Furthermore, with these measurements to find prognostic tools to predict poor functional outcome and the necessary of revision, first, we used the pre-operative CSA which assesses the possible association of implant failure due to rotator cuff failure or progressive glenoid erosion. Second, we measured the LGHO before and after surgery. Finally, with best-fit circle technique, we measured the deviation COR of the prosthetic humeral head from native anatomy after resurfacing humeral head arthroplasty.

All measurements were performed on the shoulders of patients operated for primary, end-stage gleno-humeral osteoarthritis with a Global CAP resurfacing hemiarthroplasty. The group consisted of patients who underwent a revision arthroplasty and patients with good patient reported outcome measures.

\section{Materials and methods Patient selection}

Between 2007 and 2009, 48 shoulders were operated using a Global CAP uncemented resurfacing shoulder hemiarthroplasty at two regional hospitals in the Netherlands (Alrijne Hospital and Spaarne Hospital). This cohort consisted of 12 males and 36 females. All patients were operated on by two senior orthopedic surgeons (AvN or CV) specialized in shoulder pathology. The included 48 shoulders with only primary gleno-humeral osteoarthritis had intact rotator cuff, sufficient bone stock ( $>60 \%)$ of the proximal humerus, and type A1, A2 or B1 glenoid (Walch Classification [17]) as assessed on radiographs and magnetic resonance imaging (MRI) scans. Patients with severe fatty infiltration (Goutallier [18] grade 4), paresis of rotator cuff muscles, wound healing problems, neuromuscular pathologies, or active infections were excluded for this study.

\section{Surgical protocol}

The orthopedic surgeons did not use radiological planning prior to surgery. All operations were performed via deltopectoral approach. Osteophytes present were removed, and the cartilage of the head was reamed guided by the anatomical neck of the humerus. Appropriately sized prosthesis was placed in patient own (retro) version and inclination. The prosthesis is available in five sizes, and each size has two heights to match the anatomy of the proximal humerus. No glenoid implants were used. Due to a hydroxyapatite coating, no cement was used for fixation. Digital pre- and postoperative radiographs were retrieved from the 48 shoulders. The postoperative treatment protocol was immobilization with an arm sling on the first day. Hereafter, active and passive movement supervised by a physiotherapist was allowed. After 6 weeks, free and active movement, respecting the patient's pain threshold, was encouraged and supervised by a physiotherapist.

\section{Radiographic measurements}

Radiographic measurements were performed to assess the critical shoulder angle (CSA), length of gleno-humeral offset (LGHO), and the center of rotation (COR). For reliable assessments, four independent observers performed the measurements: two senior musculoskeletal radiologists (SB and BdW) and two orthopedic surgeons (PG and JW) specialized in shoulder pathology and shoulder arthroplasty performed the measurements. All measurements were taken electronically on radiographs displayed on a PACS workstation (Cerner Corp. Kansas City, Missouri, USA). Patient characteristics and patient-reported outcomes and revisions were unknown to the assessors. The X-ray technique of the two hospitals was standardized; the patients were positioned standing with their back against the image receptor and the non-affected side was turned $35-45^{\circ}$ away from the image receptor. The affected arm was flexed $90^{\circ}$ in the elbow and the underarm was internally rotated. The angle of the beam was tilted $15-20^{\circ}$ in the cranial caudal direction and was centered toward the shoulder joint.

This "true" antero-posterior radiographs were used to perform the measurements. The assessors used the preoperative radiographs and the 6 weeks postoperative radiographs. If the 6 weeks radiographs were insufficient for assessment, the 1-year postoperative radiographs were used instead.

\section{Critical shoulder angle}

The critical shoulder angle (CSA) was assessed on all preoperative "true" antero-posterior (AP) shoulder radiographs. The angle was formed by a line connecting the superior and inferior bony margins of the glenoid and a line drawn from the inferior bony margin of the glenoid to the most lateral border of the acromion (Fig. 1.) [19]. The CSA angle is defined by three grades (Table 1).

\section{Length of the gleno-humeral offset}

The modified length of the gleno-humeral offset (LGHO) of the 48 shoulders was assessed on both pre- and postoperative "true" AP radiographs $[10,20,21]$. First, a line 


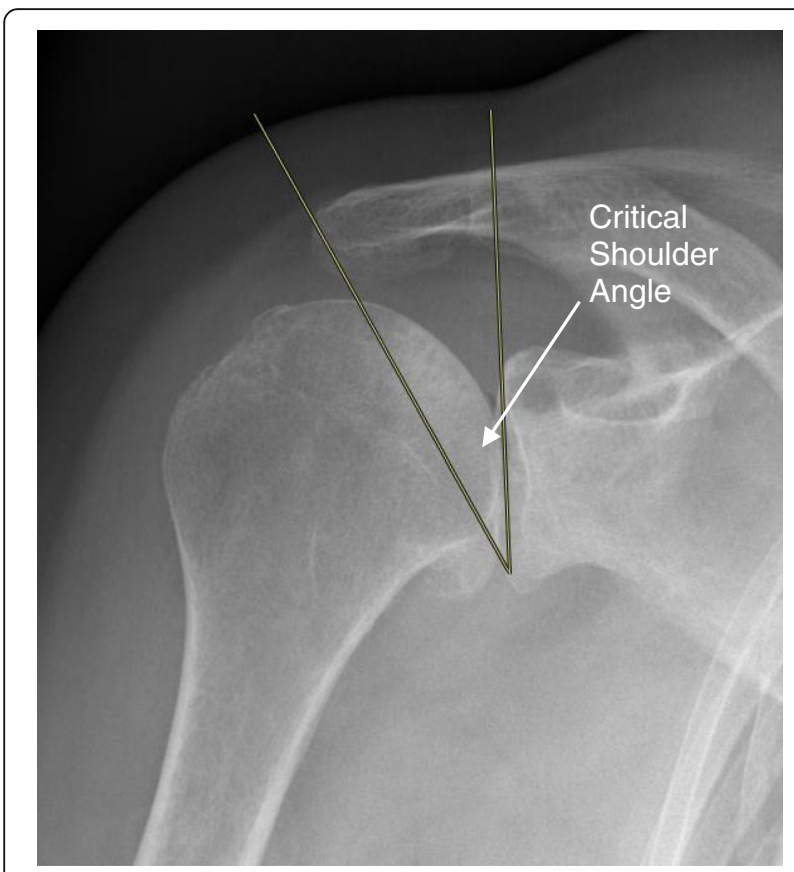

Fig. 1 Radiograph of a right shoulder showing the assessment of the critical shoulder angle

from the top to the bottom of the glenoid cavity was drawn. Second, a parallel line was drawn from the center axis of the humeral bone until the most lateral part of the greater tubercle was touched. This point was marked and the perpendicular distance from the glenoid line to this point was noted as the modified measure of LGHO (Fig. 2). The length of the gleno-humeral offset is important in shoulder function, since it affects soft tissue tension and joint balancing. Normal LGHO averages from 54 to 57 $\mathrm{mm}$ (range 43-68 $\mathrm{mm}$ ) [22]. As a result of gleno-humeral $\mathrm{OA}$, with narrowing of the joint space, the soft tissue will adapt to the changed morphology. The LGHO should not increase after surgery [10].

\section{Center of rotation}

The center of rotation (COR) was measured [8]. A best-fit circle was placed on the "true" AP radiograph using three preserved bone landmarks: the lateral cortex of the greater tubercle, medial calcar at the inflection point where calcar meets the articular surface, and the medial edge of the greater tubercle medial of the footprint of the supraspinatus tendon. A second circle, the implant matched circle, was placed to fit the curvature of the prosthetic humeral head.

Table 1 Critical shoulder angle [19]

\begin{tabular}{lll}
\hline & Angle in degrees & \\
\hline Grade I & $<30^{\circ}$ & Osteoarthritis \\
Grade II & $30^{\circ}-35^{\circ}$ & Normal \\
Grade III & $>35^{\circ}$ & Rotator cuff tear \\
\hline
\end{tabular}

The COR was identified from each circle, and the distance between the CORs was calculated in millimeter (Fig. 3.1). A coordinate system was then generated from the anatomic $\mathrm{COR}$, with the $y$-axis aligned parallel to the intramedullary axis and the $\mathrm{x}$-axis defined as perpendicular to this line. This created four regions in which the location of the decimation of COR could be defined; superior medial, inferior medial, superior lateral, and inferior lateral (Fig. 3.2). By use of the COR, we measured the overstuffing of the shoulder joint after resurfacing shoulder prosthesis. Medial deviation of the COR was defined as overstuffing [8].

\section{Revision}

At the 9-year follow-up (range 5-12 years), 12 shoulders (23\%) had a revision to a total shoulder arthroplasty. One patient had a revision for pain and loss of range of motion. On the radiographs, there was progressive glenoid erosion. At revision to total shoulder arthroplasty (TSA), the tissue samples retained per-operatively were tested positive on Pantoea agglomerans, Staphylococcus epidermidis, and Propionium acnes. We excluded this patient from data analysis for infection reason. The 11 revision patients used in data analysis are mentioned in Table 2. All other revisions had negative peroperative (tissues obtained during surgery/revision operation).

\section{Statistical analysis}

Statistical analysis was performed by use of Statistical Package for the Social Sciences (SPSS) (IBM, Armonk, NY, USA, version 26.0). After confirmation of normal distribution, continuous variables are presented as means with standard deviations (SD). Categorical data are described as frequencies with accompanying proportions. Differences between the revision and non-revision group were assessed using Student's $t$ tests or chisquared tests, where appropriate.

Inter-observer reliability was assessed by calculating of the intra-class coefficient (ICC agreement, two-way random effect model) [23]. An ICC $>0.7$ was considered as sufficient [24, 25]. Additionally, the standard error of measurement (SEM) was calculated as the square root of the withinsubject variance (i.e., sum of the between measures variance and the residual variance) with accompanying smallest detectable difference (SDD) as 1.96* $\sqrt{2} * \mathrm{SEM}[26]$.

To identify predictors for revision, univariate logistic regression was performed for potential predictors, such as age, gender, CSA, LGHO, and COR. In the case of significant association (adjusted significance level of 0.10), the factors were entered in a multivariate logistic regression analysis. For all analyses, odds ratios (OR) with 95\% confidence interval $(95 \% \mathrm{CI})$ were calculated and presented.

To calculate an optimal cut-off value of the measurement that was significantly associated with revision in the final model, a receiver operating characteristic (ROC) curve analysis 


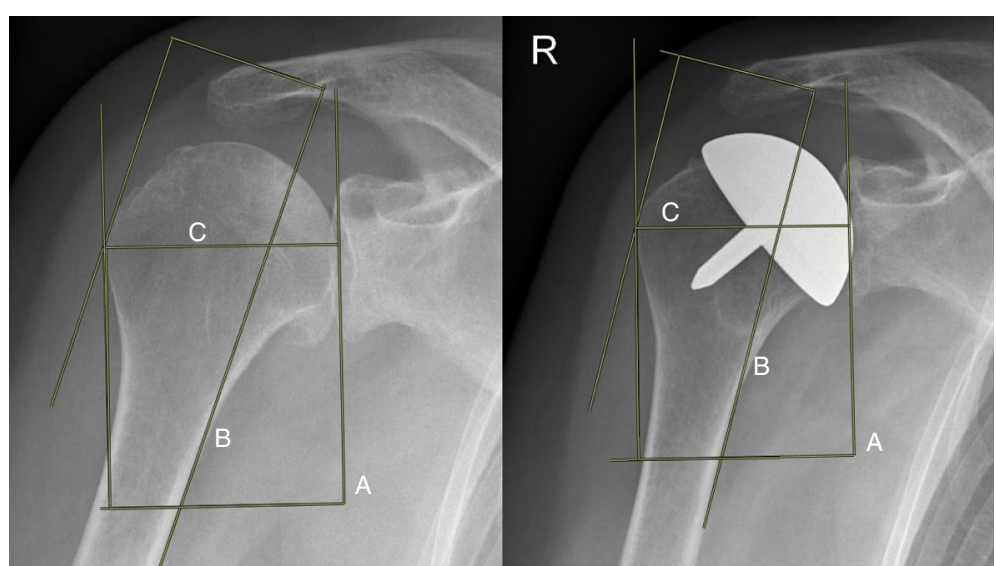

Fig. 2 Radiograph of the right shoulder before and 6 months after implantation of the resurfacing hemiarthroplasty. The modified length of gleno-humeral offset is measured by drawing a line from the top to the bottom of the glenoid (a). The cortex of the humerus was drawn. A parallel line was shifted till it touched the most lateral cortex of the greater tubercle (b). The distance was marked as (c)

was performed. A bootstrapping procedure, drawing 1000 bootstrap samples, was used to estimate a standard error to provide a $95 \% \mathrm{CI}$ around the cut-off value. As a measure of accuracy, the area under the curve (AUC) was calculated.

\section{Results}

\section{Population}

The average age of the patient population was 77 years (SD $7.5)$, and 36 patients out 47 were female (77\%). The demographics and measurements of the revision and non-revision group for the CSA, LGHO, and COR are outlined in Table 3.

\section{Reliability and measurement error}

The inter-observer reliability showed excellent reliability for the CSA, LGHO pre- and postoperative, and the COR, varying between 0.91 and 0.98 (Table 4).

\section{Critical shoulder angle}

Based on the study by Moor et al. [19], CSA values were classified into three grades: grade I CSA $<30^{\circ}$, grade II CSA $30-35^{\circ}$, and grade III CSA $>35^{\circ}$ (Table 1). The mean CSA of 47 shoulders is $29.8^{\circ}$ (SD 3.9). We found no significant difference in CSA between the revision group and non-revision group $(p=0.34)$ (Table 3$)$.

\section{Length of gleno-humeral offset}

The mean LGHO increased from $49.6 \mathrm{~mm}$ (range 37.660.4) before surgery to $52.1 \mathrm{~mm}$ (range 37.2-61.7) after surgery. The increase of the LGHO was significantly higher in the revision group compared to that in the non-revision group $(p=0.04)$. The preoperative LGHO was not significantly different between the two groups $(p=0.26)$. However, the postoperative LGHO of the
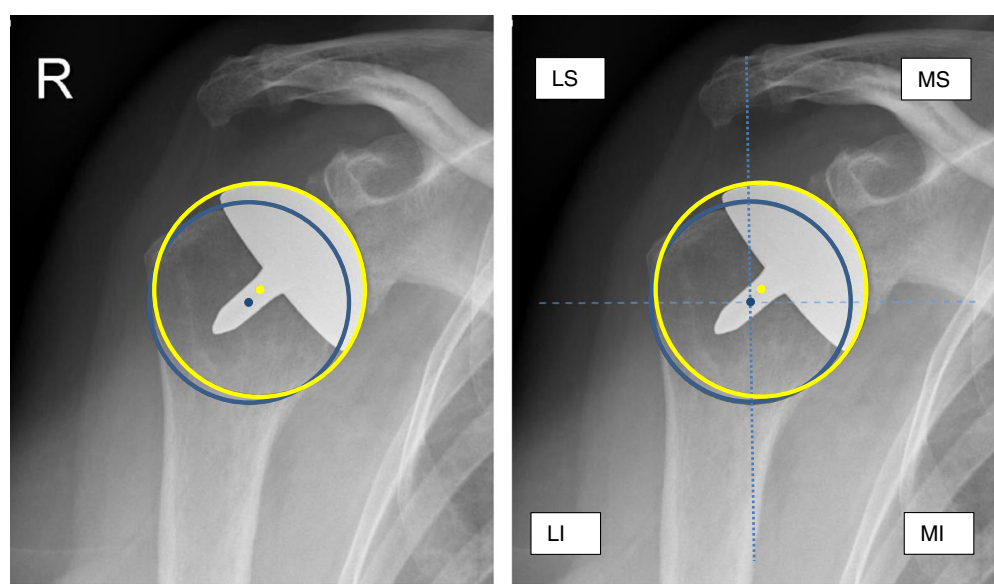

Fig. 31 Radiograph of a right shoulder. Anatomical circle with center of rotation (blue) and postoperative center of rotation (yellow). The distance in millimeters between the both centers was measured. $\mathbf{2}$ Radiograph of a right shoulder, demonstrating the anatomical circle (blue) and the implant matched circle (yellow) and the 4 quadrants. LS, lateral superior; MS, medial superior; MI, medial inferior; LI, lateral inferior 
Table 2 Revision of 11 patients

\begin{tabular}{lll}
\hline Reason & Revision & Comment \\
\hline Glenoid erosion & TSA & Progressive pain \\
Arthrofibrosis & TSA & Pain and poor function \\
Severe glenoid erosion & TSA & Progressive pain \\
Rotator cuff arthropathy & RSA & Pain and poor function, traumatic rotator cuff tear, glenoid erosion \\
Rotator cuff arthropathy & RSA & Earlier surgical subscapularis tendon repair \\
Pain and poor function & TSA & Progressive pain and loss of range of motion, minimal glenoid erosion \\
Pain and poor function & TSA & Patient is emigrated, revision surgery was abroad \\
Severe glenoid erosion & RSA & Progressive pain \\
Glenoid erosion & TSA & Progressive pain \\
Glenoid erosion & TSA & Progressive pain \\
Severe glenoid erosion & RSA & Progressive pain and loss of range of motion \\
\hline
\end{tabular}

$T S A$ total shoulder arthroplasty, RSA reverse shoulder arthroplasty

revision group was significantly different compared to the non-revision group $(p=0.03)$, see Table 3 .

\section{Center of rotation}

The mean deviation of the postoperative resurfacing head COR from the anatomic COR for all 47 cases was $5.6 \mathrm{~mm}$ (2.7 SD).

The mean COR in the non-revision and the revision group was $4.9 \mathrm{~mm}(2.5 \mathrm{SD})$ and $8.0 \mathrm{~mm}$ (SD2.2), respectively. This difference was significant $(p<0.01)$. Of the 47 shoulders, five implants (12\%) had the COR shifted to medial inferior. The remaining 43 shoulders had the COR shifted to medial superior. All shoulders in the revision group $(n=11)$ had the COR shift to medial superior, meaning overstuffing of the joint.

\section{Predictors of revision}

Univariate analysis revealed that post-operative LGHO and the COR were both significantly associated with revision. However, in the final model only, the COR remained as a predictor for revision with an OR of 1.90 (95\%Cl 1.19-3.02), see Table 5.

ROC analysis of the COR revealed a cut-off point for revision of $5.8 \mathrm{~mm}(95 \% \mathrm{Cl} 4.0-8.4)$ with a corresponding AUC of 0.82 (95\%CI 0.68-0.95).

\section{Discussion}

Inaccurate sizing or positioning of a prosthetic humeral head can lead to overstuffing the joint and poor outcomes, including glenoid erosion, rotator cuff tearing, and, in the case of a glenoid component, wear and loosening [27-32].

We assessed the CSA, LGHO, and COR in a selected cohort of patients operated on with a Global CAP, an uncemented resurfacing shoulder hemiarthroplasty for primary end-stage osteoarthritis.

Table 3 Demographics and measurements

\begin{tabular}{|c|c|c|c|c|}
\hline & Total $(n=47)$ & Revision $(n=11)$ & Non-revision $(n=36)$ & $p$ value \\
\hline Age, years, mean (SD) & $76.6(7.5)$ & $74.8(6.4)$ & $77.1(7.9)$ & 0.39 \\
\hline \multicolumn{5}{|l|}{ Gender, $n$ (\%) } \\
\hline Male & $11(23)$ & $4(36)$ & $7(19)$ & \multirow[t]{2}{*}{0.25} \\
\hline Female & $36(77)$ & $7(64)$ & $29(81)$ & \\
\hline CSA, mean (SD) & $29.8(3.9)$ & $30.8(3.0)$ & $29.5(4.2)$ & 0.34 \\
\hline \multicolumn{5}{|l|}{ CSA, $n(\%)$} \\
\hline$<30$ & $26(55)$ & $5(46)$ & $21(58)$ & \multirow[t]{3}{*}{0.66} \\
\hline $30-35$ & $16(34)$ & $5(46)$ & $11(31)$ & \\
\hline$>30$ & $5(11)$ & $1(8)$ & $4(11)$ & \\
\hline LGHO pre-operative, mean (SD) & $49.6(5.0)$ & $51.1(4.0)$ & $49.1(5.3)$ & 0.26 \\
\hline LGHO post-operative, mean (SD) & $52.1(4.9)$ & $54.9(4.4)$ & $51.3(4.8)$ & 0.03 \\
\hline LGHO CFB (SD) & $2.6(3.3)$ & $3.9(1.7)$ & $2.2(3.6)$ & 0.04 \\
\hline COR, mean (SD) & $5.6(2.7)$ & $8.0(2.2)$ & $4.9(2.5)$ & $<0.01$ \\
\hline
\end{tabular}

CSA critical shoulder angle (degrees), LGHO length of the gleno-humeral offset ( $\mathrm{mm}$ ), COR center of rotation ( $\mathrm{mm}$ ), CFB change from baseline 
Table 4 Inter-observer reliability

\begin{tabular}{lllll}
\hline & CSA & LGHO pre & LGHO post & COR \\
\hline ICC $(95 \% \mathrm{Cl})$ & $0.97(0.95-0.98)$ & $0.96(0.93-0.97)$ & $0.91(0.85-0.95)$ & $0.98(0.96-0.99)$ \\
SEM & 0.69 & 1.13 & 1.52 & 0.43 \\
SDD & 1.91 & 3.12 & 4.22 & 1.2 \\
\hline
\end{tabular}

CSA critical shoulder angle (degrees), LGHO length of the gleno-humeral offset $(\mathrm{mm})$-pre- and postoperative, COR center of rotation ( $\mathrm{mm}$ ), ICC inter-observer reliability, SEM standard error of measurement, SDD smallest detectable difference

The aim of this study was to measure inter-observer reliability of the CSA, LGHO, and COR and to define parameters to predict failure. The purpose of the Global CAP, like many other RHHI, is to recreate the normal anatomical gleno-humeral relationship of the shoulder. As considered by Mechlenburg et al. [10] and Alolabi et al. [8], the RHHI might potentially overstuff the gleno-humeral joint.

We found a high inter-observer reliability for the CSA, this is in line with other studies on CSA measurements [33]. Moor et al. [19] classified a CSA angle $<30^{\circ}$ as gleno-humeral $\mathrm{OA}$ and a CSA $>35^{\circ}$ as rotator cuff tear. In our series, with the observed minimal detectable difference of $1.9^{\circ}$, this classification should be interpreted with caution. Viehöfer et al. [34] showed that a higher CSA requires more rotator cuff activity to preserve joint stability. This leads to higher risk of rotator cuff failure [35-37]. Additionally, Watling et al. [38] found a high CSA being associated with glenoid component loosening and failure. In our series, however, we did not find a significant association between CSA angles and revision.

Originally, the measurements of the LGHO is performed using the distance from the base of the coracoid process to greater tubercle [31, 39]. But this measure shows systematic errors in inter-tester reliability because it is difficult to locate the base of the coracoid process [31]. Due to the reported problems with inter-tester

Table 5 Predictors of revision

\begin{tabular}{lll}
\hline Univariate & OR $(95 \% \mathrm{Cl})$ & $p$ value \\
Age & $0.96(0.87-1.05)$ & 0.38 \\
Gender & $2.37(0.54-10.40)$ & 0.25 \\
CSA & $1.09(0.92-1.30)$ & 0.33 \\
LGHO preoperative & $1.09(0.94-1.25)$ & 0.26 \\
LGHO postoperative & $1.19(1.01-1.41)$ & 0.04 \\
LGHO change from baseline & $1.19(0.94-1.49)$ & 0.15 \\
COR & $1.90(1.19-3.02)$ & 0.01 \\
Multivariate & OR (95\%Cl) & $p$ value \\
LGHO postoperative & $1.16(0.95-1.43)$ & 0.15 \\
COR & $1.91(1.14-3.20)$ & 0.02 \\
Final model & OR (95\%Cl) & $p$-value \\
COR & $1.90(1.19-3.02)$ & 0.01 \\
\hline
\end{tabular}

CSA critical shoulder angle, $L G H O$ length of the gleno-humeral offset, COR center of rotation, $O R$ odds ratio reliability of the standard LGHO measurements, we used the modified LGHO [10, 20, 21]. Because, it is possible that factors like direct postoperative intra-articular fluid or releases related capsular laxity might falsely increase the LGHO measurements, we used the 6 weeks or 1year post-operative radiographs.

In theory, LGHO after surgery should be identical to LGHO before the shoulder morphology changed caused by arthritis without structural changes of the soft tissue. But as osteoarthritis progresses with narrowing of the joint space, destruction of the joint cartilage, and capsule tightening, the soft tissue adapts to the changed morphology by losing elasticity and the LGHO should not be increased after surgery $[10,20,21]$. This in contrast with current study where the mean change of baseline of the LGHO increased by $2.6 \mathrm{~mm}$ and $3.9 \mathrm{~mm}$ in the nonrevision group and revision group, respectively.

Like Mechlenburg et al. [10] in our study the LGHO is not reproduced. Additionally, the difference between the postoperative LGHO between the revision and nonrevision is significant $(p=0.03)$. Nonetheless, we found that the postoperative LGHO is not a predictor of revision. Conform the study by Stilling et al. [21], we found high inter-observer agreement.

Alolabi et al. [8] found in their study that $65.1 \%$ of the RHHI demonstrated an inadequate reaming of the humeral head, resulting in overstuffing of the glenohumeral joint. In our study, we found $88 \%$ overstuffing in all shoulders and $100 \%$ overstuffing in the revision group.

Multiple studies use different cut-off points to define overstuffing of the gleno-humeral joint [8, 27-30, 32].

In these studies, they assessed no relation to an increase of COR to patient-reported outcomes or revision. Pearl et al. [40, 41] already showed in their computer simulation studies that the COR in RHHI have great difficulty matching the geometric dimensions of the native gleno-humeral anatomy. However, these measurements were done on cadaveric humerus, without relation to patient-reported outcomes or revision. And computer studies may not be directly comparable to the results of radiographic studies. Our results regarding RHHI are in line with Alolabi et al. [8], the normal gleno-humeral anatomy, regarding the COR, is not reproduced. We found a significant increase in COR in the revision group compared to the non-revision group. In other 
words, the probability of revision increases significantly with an increased COR. Overstuffing has always been a suspect for failure [8]. However, this has not been demonstrated in the literature before. In this current study, we have shown a relation between failure and overstuffing.

The main limitation of this study is the small study group. Because of the fact that this concerns to an ongoing study of the Global CAP, it provides valuable information of this uncemented RHHI. The rate of revision (23\%) at 9 years follow-up in our cohort is high. We excluded the patient with low-grade infection for data analysis because the authors believe it is difficult to distinguish between pain caused by glenoid erosion or pain caused by low-grade infection.

Three questions arise why the rate of revision was higher compared by studies by Levy et al. [1, 2, 42, 43]. First, a number of revisions can happen when inexperienced surgeons perform few procedures. However, the surgeons in this cohort are specialized shoulder surgeons, in high volume shoulder hospitals, with experience in shoulder replacement/revision, shoulder arthroscopic procedures, and fracture osteosynthesis.

Second, in this current study, the RHHI was positioned freehand based on anatomic landmarks, advised by the implant manufacturer, without a digital preoperative planning. The authors agree with Alolabi et al. [8], intraoperative fluoroscopy may provide additional valuable information to confirm offset and varus/valgus of the implant. Finally, explanation could be patient selection, as some patients may have benefited more with a total shoulder arthroplasty.

Another limitation to this cohort study is the use of the "true" antero-posterior radiograph of the shoulder. Theoretically, the measurements could vary according the position of the arm or the scapula. Therefore, we only used the best 6 -months or 1-year radiographs for postoperative measurements, which had better quality compared to direct postoperative radiographs. Moreover, Spiegl et al. [44] and Bouaicha et al. [45] showed that the CSA assessed on radiographs is equal to a computer tomography (CT) scan and superior to a MRI scan.

The modified LGHO was assessed in multiple studies on radiographs $[10,20,21]$; in literature, there is no study which compared the (modified) LGHO on radiographs compared to CT or MRI scan.

Many studies use the COR for hemi- and TSP arthroplasty on patient radiographs $[8,46-48]$. Other studies used CT on cadaveric shoulders to assess the COR $[40,49,50]$. In literature, we found no superior evidence for CT or radiographs.

\section{Conclusion}

In this study, we demonstrated that the CSA, LGHO, and $\mathrm{COR}$ are reliable radiologic measurement methods with high inter-observer agreement. The Global CAP resurfacing shoulder hemiarthroplasty will lead to overstuffing of the gleno-humeral joint in almost all shoulders. In contrast with the CSA and LGHO, we found a correlation between clinical failure and revision surgery in case of a deviation of the COR greater of $5 \mathrm{~mm}$.

\section{Abbreviations \\ AUC: Area under the curve; CAP: Conservative anatomic prosthesis; CFB: Change from baseline; Cl: Confidence interval; COR: Center of rotation; CSA: Critical shoulder angle; CT: Computer tomogram; ICC: Intra-class coefficient; LGHO: Length of the gleno-humeral offset; MRI: Magnetic resonance imaging; OR: Odds ratio; $\mathrm{RHHI}$ : Resurfacing humeral head implant; ROC: Receiver operating characteristic; RSA: Reverse shoulder arthroplasty; SD: Standard deviations; SDD: Smallest detectable difference; SEM: Standard error of measurement; SPSS: Statistical package for the social sciences; TSA: Total shoulder arthroplasty}

\section{Acknowledgements}

The authors are very grateful to B van der Heijden MSc (statistician) for his statistical assistance and to SM Bollen MD and BWK de Wit MD (musculoskeletal radiologists) for their valuable contribution to the measurements.

\section{Authors' contributions}

PG contributed to the study design, performed the measurements and data analysis, and wrote the manuscript. JW performed the measurements and wrote the manuscript. IS contributed to the data analysis and interpretation and writing of the manuscript. CV contributed to the study design, data analysis, and writing of the manuscript. AvN contributed in the study design, data analysis, and writing of the manuscript. All authors have read the manuscript and approved this work for publication. All authors believe that the manuscript represents honest work. We believe that there is no duplication on the internet.

\section{Funding}

This study was funded by a grant (Spaarne Gasthuis \#116347 and Alrijne Hospital \#221090) from DePuy/Synthes, Warsaw, IN, USA, which participated in the study design and data management. The implant used in this study was not provided free of charge.

The study sponsors had no role in the in the collection, analysis, and interpretation of data and in the decision to submit the manuscript for publication.

\section{Availability of data and materials}

The datasets during and/or analyzed during the current study available from the corresponding author on reasonable request.

\section{Ethics approval and consent of participate}

Trail registrations number: Institutional review board, number: ACLU 2016.0054, Ethical Committee number: CBP M1330348. Registered 7 November 2006.

All patients signed informed consent.

\section{Competing interests}

Pieter C Geervliet, the author, their immediate family, and any research foundation with which they are affiliated have not received any financial payments or other benefits from any commercial entity to subject of this article.

Jore $\mathrm{H}$ Willems, the author, their immediate family, and any research foundation with which they are affiliated have not received any financial payments or other benefits from any commercial entity to subject of this article.

Inger N Sierevelt, the author, their immediate family, and any research foundation with which they are affiliated have not received any financial payments or other benefits from any commercial entity to subject of this article.

Cornelis PJ Visser, Dr. Visser is a key opinion leader for Johnson and Johnson. Arthur van Noort, Dr. Van Noort, is a key opinion leader for Johnson and Johnson. 


\section{Author details}

'NoordWest Clinics, Department of Orthopedic Surgery, Shoulder Unit, Wilhelminalaan 12, 1815, JD, Alkmaar, the Netherlands. ${ }^{2}$ Spaarne Hospital, Spaarnepoort 1, 2134, TM, Hoofddorp, the Netherlands. ${ }^{3}$ Alrijne Hospital, Simon Smitweg 1, 2353, GA, Leiderdorp, the Netherlands.

Received: 26 October 2019 Accepted: 17 December 2019

Published online: 30 December 2019

\section{References}

1. Levy O, Funk L, Sforza G, Copeland SA. Copeland surface replacement arthroplasty of the shoulder in rheumatoid arthritis. J Bone Joint Surg Series A. 2004:86:512-8.

2. Levy O, Copeland SA. Cementless surface replacement arthroplasty (Copeland CSRA) for osteoarthritis of the shoulder. J Shoulder Elb Surg. 2004;13(3):266

3. Thomas SR, Wilson AJ, Chambler A, Harding I, Thomas M. Outcome of Copeland surface replacement shoulder arthroplasty. J Shoulder Elb Surg. 2005;14(5):485

4. Mullett H, Levy O, Raj D, Even T, Abraham R, Copeland SA. Copeland surface replacement of the shoulder: results of an hydroxyapatite-coated cementless impant in patients over 80 years of age. J Bone and Joint Surg British Volume. 2007;89(11):1466.

5. Pritchett JW. Long-term results and patient satisfaction after shoulder resurfacing. J Shoulder Elb Surg. 2011;20(5):771.

6. Al-Hadithy N, Domos P, Sewell MD, Naleem A, Papanna MC, Pandit R. Cementless surface replacement arthroplasty of the shoulder for osteoarthritis: results of fifty mark III Copeland prosthesis from an independent center with four-year mean follow-up. J Shoulder Elb Surg. 2012;21:1776-81.

7. Rasmussen JV, Polk A, Sorensen AK, Olsen BS, Brorson S. Outcome, revision rate and indication for revision following resurfacing hemiarthroplasty for osteoarthritis of the shoulder: 837 operations reported to the Danish shoulder arthroplasty registry. Bone Joint J. 2014;96:519-25.

8. Alolabi B, Youderian AR, Napolitano L, Szerlip BW, Evans PJ, Nowinski RJ, et al. Radiographic assessment of prosthetic humeral head size after anatomic shoulder arthroplasty. J Shoulder Elb Surg. 2014;23(11):1740.

9. Copeland S. The continuing development of shoulder replacement: "Reaching the surface". J Bone Joint Surg - Series A. 2006;88(4):900.

10. Mechlenburg I, Amstrup A, Klebe T, Jacobsen SS, Teichert G, Stilling M. The Copeland resurfacing humeral head implant does not restore humeral head anatomy. A retrospective study. Arch Orthop Trauma Surg. 2013;133:615-9.

11. Smith T, Gettmann A, Wellmann M, Pastor F, Struck M. Humeral surface replacement for osteoarthritis. Acta Orthop. 2013;84:468-72.

12. Alizadehkhaiyat $O$, Kyriakos A, Singer MS, Frostick SP. Outcome of Copeland shoulder resurfacing arthroplasty with a 4-year mean follow-up. J Shoulder Elb Surg. 2013;22:1352-8.

13. Geervliet PC, van den Bekerom MPJ, Spruyt P, Curvers M, van Noort A, Visser CPJ. Outcome and revision rate of uncemented glenohumeral resurfacing (C.A.P.) after 5-8 years. Archives of Orthopaedic and Trauma Surgery. 2017. Springer Berlin Heidelberg; [Cited 2017 Apr 22];1-8. Available from: http:// link.springer.com/10.1007/s00402-017-2688-9

14. Geervliet $P$, Van Den Bekerom $M$, Spruyt $P$, Curvers $M$, Visser $C$, van Noort $A$. Short-term results of the global C.A.P. uncemented resurfacing shoulder prosthesis. Orthopedics. 2014;37:42-7.

15. Engelsma Y, Buma $P$, Geervliet PC, Noort AV. Septic failure is not a septic loosening: a case report of a failed shoulder prosthesis. I J Shoulder Surg. 2012;6:1.

16. Geervliet PC, Houtveen M, Sierevelt IN, Visser CPJ, van Noort A. Revision of failed resurfacing hemiarthroplasty: midterm results, survival, and group comparison. Orthopedics. 2018;1:1-7 Available from: https://www.healio. com/doiresolver?doi=10.3928/01477447-20181206-02.

17. Walch G, Badet R, Boulahia A, Khoury A. Morphologic study of the glenoid in primary glenohumeral osteoarthritis. J Arthroplast. 1999;14:756-60.

18. Goutallier D, Postel JM, Bernageau J, Lavau L, Voisin MC. Fatty infiltration of disrupted rotator cuff muscles. Rev Rhum. 1995;62:415-22 Available from: http://www.ncbi.nlm.nih.gov/pubmed/7552205.

19. Moor BK, Bouaicha S, Rothenfluh DA, Sukthankar A, Gerber C. Is there an association between the individual anatomy of the scapula and the development of rotator cuff tears or osteoarthritis of the glenohumeral joint? A radiological study of the critical shoulder angle. Bone Joint J. 2013; 95(7):935

20. Mechlenburg I, Klebe TM, Døssing KV, Amstrup A, Søballe K, Stilling M Evaluation of periprosthetic bone mineral density and postoperative migration of humeral head resurfacing implants: two-year results of a randomized controlled clinical trial. J Shoulder Elb Surg. 2014;23:1427-36 Available from: https://linkinghub.elsevier.com/retrieve/pii/S1058274614002730.

21. Stilling M, Mechlenburg I, Amstrup A, Soballe K, Klebe T. Precision of novel radiological methods in relation to resurfacing humeral head implants: assessment by radiostereometric analysis, DXA, and geometrical analysis Arch Orthop Trauma Surg. 2012;132:1521-30 Available from: http://link. springer.com/10.1007/s00402-012-1580-X.

22. Iannotti JP, Gabriel JP, Schneck SL, Evans BG, Misra S. The normal glenohumeral relationships. An anatomical study of one hundred and forty shoulders. J Bone Joint Surg - Series A. 1992;74(4):491.

23. de Vet HCW, Terwee CB, Knol DL, Bouter LM. When to use agreement versus reliability measures. J Clin Epidemiol. 2006:59(10):1033.

24. Streiner DL, Norman GR. Health measurement scales: a practical guide to their development and use. Health measurement scales: a practical guide to their development and use. USA: Oxford University Press; 2008.

25. Weir JP. Quantifying test-retest reliability using the intraclass correlation coefficient and the SEM. J Strength Cond Res. 2005;19(1):231.

26. de Vet HC, Terwee CB, Ostelo RW, Beckerman H, Knol DL, Bouter LM. Minimal changes in health status questionnaires: distinction between minimally detectable change and minimally important change. Health Qual Life Outcomes. 2006;4(1):54.

27. Favre P, Moor B, Snedeker JG, Gerber C. Influence of component positioning on impingement in conventional total shoulder arthroplasty. Clin Biomech. 2008;23(2):175

28. Harryman DT, Sidles JA, Harris SL, Lippitt SB, Matsen FA. The effect of articular conformity and the size of the humeral head component on laxity and motion after glenohumeral arthroplasty: a study in cadavera. J Bone Joint Surg - Series A. 1995;77(4):555

29. Nyffeler RW, Sheikh R, Jacob HAC, Gerber C. Influence of humeral prosthesis height on biomechanics of glenohumeral abduction: an in vitro study. $J$ Bone Joint Surg - Series A. 2004;77(4):555.

30. Terrier A, Ramondetti S, Merlini F, Pioletti DD, Farron A. Biomechanical consequences of humeral component malpositioning after anatomical total shoulder arthroplasty. J Shoulder Elb Surg. 2010;19(8):1184.

31. Thomas SR, Sforza G, Levy O. Copeland SA. Geometrical analysis of Copeland surface replacement shoulder arthroplasty in relation to normal anatomy. J Shoulder Elb Surg. 2005;14(2):186.

32. Williams GR, Wong KL, Pepe MD, Tan V, Silverberg D, Ramsey ML, et al. The effect of articular malposition after total shoulder arthroplasty on glenohumeral translations, range of motion, and subacromial impingement. J Shoulder Elb Surg. 2001;10(5):399.

33. Hussami M, Goetti P, Terrier A, Farron A, Omoumi P, Becce F. Annual Scientific Meeting Abstracts of the European Society of Musculoskeletal Radiology (ESSR) 2018, Amsterdam, The Netherlands. Skelet Radiol. 2018;47: 895-909 Available from: http://link.springer.com/10.1007/s00256-018-2924-6.

34. Viehöfer AF, Gerber C, Favre P, Bachmann E, Snedeker JG. A larger critical shoulder angle requires more rotator cuff activity to preserve joint stability. J Orthop Res. 2016;34:961-8 Available from: http://doi.wiley.com/10.1002/jor.23104.

35. Moor BK, Kuster R, Osterhoff G, Baumgartner D, CML W, Zumstein MA, et al. Inclination-dependent changes of the critical shoulder angle significantly influence superior glenohumeral joint stability. Clin Biomech. 2016;32:268.

36. Gerber C, Snedeker JG, Baumgartner D, Viehöfer AF. Supraspinatus tendon load during abduction is dependent on the size of the critical shoulder angle: a biomechanical analysis. J Orthop Res. 2014;32(7):952.

37. Mantell MT, Nelson R, Lowe JT, Endrizzi DP, Jawa A. Critical shoulder angle is associated with full-thickness rotator cuff tears in patients with glenohumeral osteoarthritis. J Shoulder Elb Surg. 2017;26(12):e376.

38. Watling JP, Sanchez JE, Heilbroner SP, Levine WN, Bigliani LU, Jobin CM. Glenoid component loosening associated with increased critical shoulder angle at midterm follow-up. J Shoulder Elb Surg. 2018;27(3):449.

39. Lehtinen JT, Belt EA, Kauppi MJ, Kaarela K, Kuusela PP, Kautiainen HJ, et al. Bone destruction, upward migration, and medialisation of rheumatoid shoulder: a 15 year follow up study. Ann Rheum Dis. 2001;60(4):322.

40. Pearl ML, Kurutz S, Robertson DD, Yamaguchi K. Geometric analysis of selected press fit prosthetic systems for proximal humeral replacement. J Orthop Res. 2002;20(2):192 
41. Pearl ML, Kurutz S. Geometric analysis of commonly used prosthetic systems for proximal humeral replacement. J Bone Joint Surg (Am Vol). 1999;81(5):660

42. Levy O, Tsvieli O, Merchant J, Young L, Trimarchi A, Dattani R, et al. Surface replacement arthroplasty for glenohumeral arthropathy in patients aged younger than fifty years: results after a minimum ten-year follow-up. Journal of shoulder and elbow surgery. Elsevier Ltd. 2015;24:1049-60.

43. Levy O, Copeland S. Cementless surface replacement arthroplasty of the shoulder: 5- to 10-year results with the Copeland mark-2 prosthesis. J Bone Joint Surg Br Vol. 2001;83(2):213.

44. Spiegl UJ, Horan MP, Smith SW, Ho CP, Millett PJ. The critical shoulder angle is associated with rotator cuff tears and shoulder osteoarthritis and is better assessed with radiographs over MRI. Knee Surg Sports Traumatol Arthrosc. 2016;24(7):2244.

45. Bouaicha S, Ehrmann C, Slankamenac K, Regan WD, Moor BK. Comparison of the critical shoulder angle in radiographs and computed tomography. Skelet Radiol. 2014;43(8):1053.

46. Youderian AR, Ricchetti ET, Drews M, lannotti JP. Determination of humeral head size in anatomic shoulder replacement for glenohumeral osteoarthritis. J Shoulder Elb Surg. 2014;23(7):955

47. von Engelhardt LV, Manzke M, Breil-Wirth A, Filler TJ, Jerosch J. Restoration of the joint geometry and outcome after stemless TESS shoulder arthroplasty. World J Orthop. 2017;8(10):790.

48. Chalmers PN, Granger EK, Orvets ND, Patterson BM, Chamberlain AM, Keener JD, et al. Does prosthetic humeral articular surface positioning associate with outcome after total shoulder arthroplasty? J Shoulder Elb Surg. 2018;27(5):863.

49. Pearl ML, Kurutz S, Postachini R. Geometric variables in anatomic replacement of the proximal humerus: how much prosthetic geometry is necessary? J Shoulder Elb Surg. 2009;18(3):366.

50. Jeong J, Bryan J, lannotti JP. Effect of a variable prosthetic neck-shaft angle and the surgical technique on replication of normal humeral anatomy. J BoneJoint Surg - Series A. 2009;91(8):1932.

\section{Publisher's Note}

Springer Nature remains neutral with regard to jurisdictional claims in published maps and institutional affiliations.

Ready to submit your research? Choose BMC and benefit from:

- fast, convenient online submission

- thorough peer review by experienced researchers in your field

- rapid publication on acceptance

- support for research data, including large and complex data types

- gold Open Access which fosters wider collaboration and increased citations

- maximum visibility for your research: over $100 \mathrm{M}$ website views per year

At $\mathrm{BMC}$, research is always in progress.

Learn more biomedcentral.com/submissions 